\title{
A Phase II Trial of Osimertinib in the Second-Line Treatment of Non-small Cell Lung Cancer with the EGFR T790M Mutation, Detected from Circulating Tumor DNA: LiquidLung-0-Cohort 2
}

\author{
Cheol-Kyu Park, MD, PhD' \\ Hyun-Ju Cho, MS ${ }^{1}$ \\ Yoo-Duk Choi, MD, PhD² \\ In-Jae Oh, MD, $P h D^{1}$ \\ Young-Chul Kim, MD, PhD'
}

${ }^{1}$ Department of Internal Medicine, Chonnam National University Hwasun Hospital, Hwasun, ${ }^{2}$ Department of Pathology, Chonnam National University Medical School, Gwangju, Korea

\begin{abstract}
Purpose
Administering the best treatment after failure of epidermal growth factor receptor (EGFR) tyrosine kinase inhibitor (TKI) therapy requires knowledge of resistance status. In this trial, treatment efficacy of osimertinib was assessed in patients with non-small cell lung carcinoma (NSCLC) harboring the T790M resistance mutation, detected from circulating tumor DNA (ctDNA) with unknown tumor mutation status.
\end{abstract}

\section{Materials and Methods}

To extract ctDNA from plasma, $15 \mathrm{~mL}$ of peripheral blood was withdrawn and centrifuged immediately before storage. Cobas ver. 2 and PANA Mutyper were used for ctDNA genotyping. Patients with T790M, detected from ctDNA, were enrolled and they received a oncedaily administration of osimertinib $80 \mathrm{mg}$. The primary endpoint was objective response rate (ORR), and secondary endpoints were ctDNA test sensitivity, progression-free survival (PFS), duration of response (DoR), and safety.

\section{Results}

Eighty patients with acquired resistance to prior EGFR-TKI therapies were screened. ctDNA of 21 patients showed T790M positivity, and 19 patients were enrolled. In the responseevaluable population ( $n=15)$, ORR was $66.7 \%$ (10/15). Median PFS was 8.3 months (95\% confidence interval [Cl], 7.9 to 8.7) and median DoR was 6.8 months (95\% Cl, 5.3 to 8.3) in the intent-to-treat population $(n=19)$. No subject experienced drug-related adverse event of grades $\geq 3$ or required dose reduction. The sensitivity of the ctDNA tests was $56.8 \%$ using both methods and $45.9 \%$ with either method from the estimated T790M-positive cases.

\section{Conclusion}

Osimertinib has favorable efficacy in patients with NSCLC harboring T790M, detected from ctDNA with unknown tumor mutation status, in whom disease had progressed during prior EGFR-TKI therapy.

\section{Tel: 82-61-379-7614}

Fax: 82-61-379-7619

E-mail: kyc0923@jnu.ac.kr

Received July 4, 2018

Accepted September 5, 2018

Published Online September 7, 2018

*These data were presented at the World

Conference of Lung Cancer, Yokohama, 2017.
Key words

Osimertinib, T790M, Circulating tumor DNA,

Non-small cell lung carcinoma

\section{Introduction}

Resistance develops in most patients treated with epidermal growth factor receptor-tyrosine kinase inhibitors (EGFRTKIs) as first-line treatment for EGFR-mutant non-small cell lung cancer (NSCLC). Among several resistance mechanisms, the EGFR T790M mutation occurs in up to $60 \%$ of these patients [1].
Osimertinib is an orally bioavailable, potent, and irreversible third-generation EGFR-TKI for EGFR sensitizing mutations, including the T790M resistance mutation, and has a significant selectivity margin against wild-type EGFR [2-5]. Studies of osimertinib (AURA series) in patients with T790Mpositive NSCLC who were previously treated with EGFRTKI have shown promising efficacy with osimertinib [2-4]. In a tissue-based assay, the objective response rate (ORR) ranged from $62 \%$ to $71 \%$, the median duration of response 


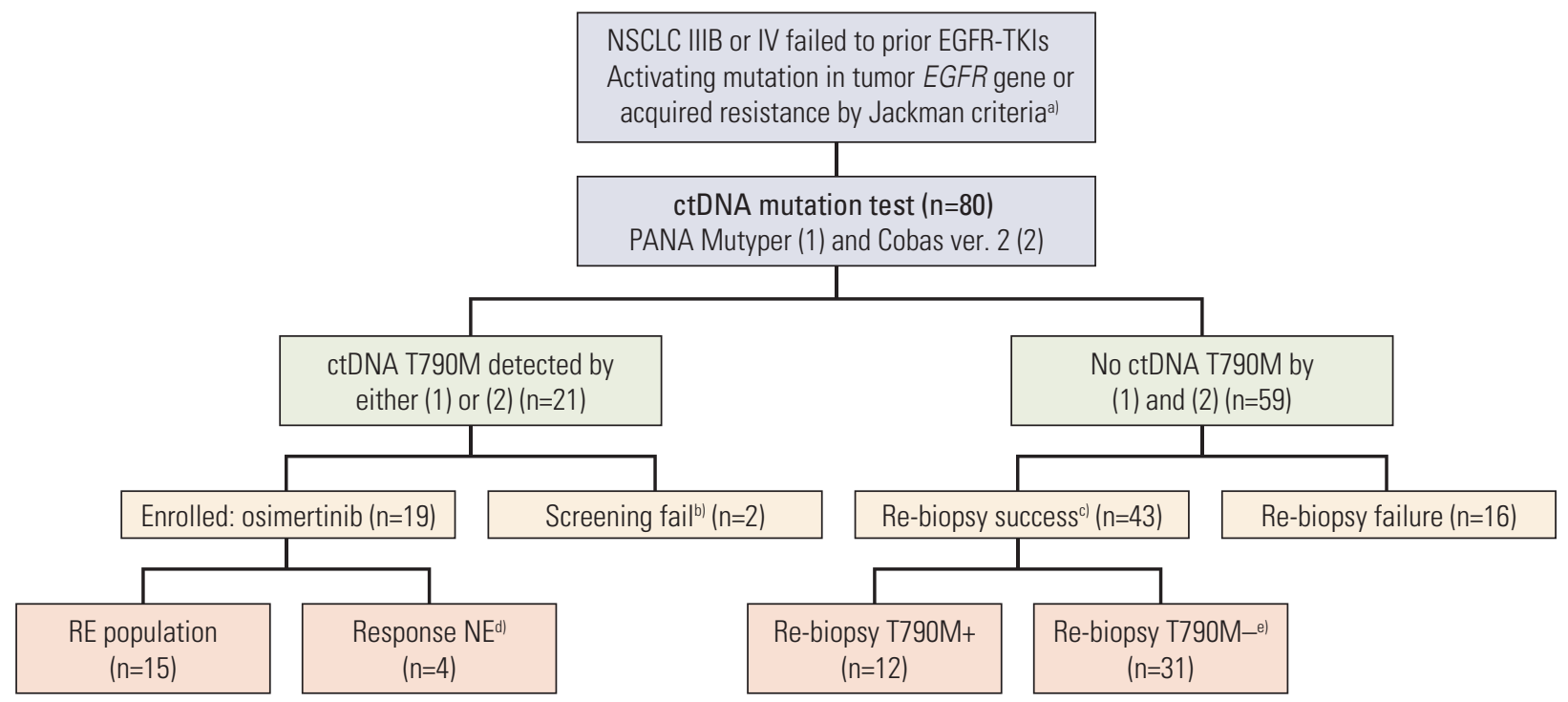

Fig. 1. Study subjects screened and enrolled, diagnostic study assignments, and exclusions. NSCLC, non-small cell lung cancer; EGFR-TKIs, epidermal growth factor receptor-tyrosine kinase inhibitors; ctDNA, circulating tumor DNA; RE, response evaluable; NE, not evaluable. ${ }^{a}$ Must have experienced clinical benefit from prior EGFR-TKI, according to the Jackman criteria, followed by systemic objective progression (RECIST) while on continuous treatment with EGFR-TKI, berformance deterioration $(n=1)$, expire $(n=1)$, 'Tumor tissue $(n=35)$, pleural fluid cytology $(n=5)$, cerebrospinal fluid cytology $(\mathrm{n}=2)$, and fine needle aspiration cytology $(\mathrm{n}=1)$, ${ }^{\mathrm{d}}$ Response not evaluable: poor adherence to treatment due to sepsis $(\mathrm{n}=1)$ and rapid deterioration leading to death $(n=3)$, ${ }^{e}$ Negative $(n=28)$, invalid or not requested for analysis $(n=3)$.

(DoR) ranged from 9.7 to 15.2 months, and the median progression-free survival (PFS) ranged from 9.6 to 12.3 months. In addition, osimertinib has acceptable toxicity and tolerability compared with the first- and second-generation EGFRTKIs [2-4]. As a result, osimertinib is currently being applied in real practice, or is under investigation, as a treatment option in: (1) patients with advanced T790M-positive NSCLC who have previously failed an EGFR-TKI therapy; (2) patients with advanced EGFR-mutant NSCLC who are treatment-naïve [5]; or (3) combination with novel agents for patients with EGFR-TKI-resistant NSCLC [6].

The significant evidence of osimertinib efficacy is based on studies in patients with EGFR T790M mutations detected from tumor DNA. Thus, the knowledge of EGFR mutation status is a crucial step in selecting the best treatment in firstand second-line settings after acquiring resistance. Although tumor biopsy is the conventional, standard method for mutation detection, obtaining sufficient tumor tissue or cytology samples for analysis remains a challenge [7], especially from patients in whom the disease progressed after previous therapy. Recent studies have shown that circulating tumor DNA (ctDNA) can be used as a suitable substitute for tumor DNA analysis of the EGFR T790M mutation and activating mutation statuses [8-10]. ctDNA from peripheral blood can track the genomic evolution of tumor cells and reflect the genetic landscape of metastatic and primary tumors [7]. Plasma genotyping using ctDNA has been demonstrated as a highly sensitive and specific technique for detection of the EGFR mutation, with an excellent positive predictive value [9].

Promising evidence of the efficacy of osimertinib treatment has also been observed in patients who are positive for the T790M mutation detected in plasma $[4,11,12]$ (ORR $64 \%$ to $77 \%$ and PFS 8.2 to 9.3 months), suggesting that liquid biopsy using plasma ctDNA provides an alternative when tumor biopsy is not feasible for identifying patients who may benefit from third-generation EGFR-TKI, with almost equivalent efficacy to tissue analysis. However, there has been no prospective trial showing the efficacy of osimertinib in patients who tested positive for T790M mutation on plasma analysis.

Based on unmet clinical needs, this trial assessed the treatment efficacy of osimertinib in patients with NSCLC harboring the T790M mutation, which was detected from ctDNA with unknown tumor EGFR mutation status, after failure of previous EGFR-TKI. 


\section{Materials and Methods}

\section{Trial design and subjects}

This was a phase II, open-label, single-center study of osimertinib in patients with locally advanced or metastatic NSCLC in whom the disease had progressed after prior EGFR-TKI (gefitinib, erlotinib, afatinib, or dacomitinib) therapy. Between September 2016 and September 2017, we recruited patients with histologically or cytologically-diagnosed NSCLC harboring activating EGFR mutations (G719X, exon 19 deletion, L858R, L861Q) or patients who had experienced clinical benefits from prior EGFR-TKI therapy, followed by systemic objective progression while on continuous treatment with EGFR-TKIs according to the Jackman criteria [13].

All patients were required to provide a blood sample at screening to test for the T790M mutation in plasma. Fifteen $\mathrm{mL}$ of peripheral blood was withdrawn and centrifuged immediately before storage. ctDNA extraction and EGFR mutation analysis were performed by PANA Mutyper (Panagene Inc., Daejeon, Korea, hereafter Pana), based on peptide nucleic acid (PNA)-mediated polymerase chain reaction (PCR) clamping and melting technology, and the Cobas EGFR mutation test, ver. 2 (Roche Molecular Systems, Pleasanton, CA, hereafter Cobas), based on a real-time PCR technique, simultaneously. Patients who harbored the T790M mutation, detected by either of the two tests, with unknown tumor EGFR mutation status were enrolled in this trial. If the T790M mutation was not detected by both tests, re-biopsy was recommended and attempted in feasible patients. For genotyping of tumor tissue or cytology samples, the PNA Clamp EGFR mutation detection kit (Panagene Inc.) was used (Fig. 1).

Eligibility criteria are provided in detail in the Supplementary Material. Patients with central nervous system (CNS) metastases could be enrolled if their disease was asymptomatic or stable after local therapy, including surgery or radiotherapy, before the first dose of osimertinib.

\section{Trial procedure, assessment, and treatment}

Eligible patients received $80 \mathrm{mg}$ of osimertinib once a day, regardless of food ingestion, and treatment continued until disease progression as defined by the Response Evaluation Criteria in Solid Tumors (RECIST) ver. 1.1 [14], unacceptable toxicity, or another reason occurred. Response evaluation was performed every 8 weeks for the first three assessments (24 weeks), followed by every 12 weeks, for subsequent assessments. Brain imaging studies were regularly performed in patients with known brain metastases. For the remaining patients, brain imaging studies were performed when they showed symptoms or signs of CNS metastases. Patients could continue osimertinib after RECIST 1.1-defined progression if they maintained clinical benefits, as assessed by an investigator.

The primary objective was ORR (assessed by RECIST 1.1) of osimertinib in NSCLC after acquired resistance to prior EGFR-TKI, with the T790M mutation detected from ctDNA. The secondary objective was to compare the sensitivity of the EGFR mutation detection methods (Pana vs. Cobas) using ctDNA. In addition, PFS, DoR, and safety and tolerability parameters of osimertinib were also measured. PFS was defined as the time (in months) from the first date of osimertinib treatment until the date of objective disease progression or death, regardless of whether the patient was withdrawn from therapy or received another anticancer therapy prior to progression. DoR was defined as the time (in months) from the documentation of the tumor response to disease progression or death in a patient who had the best overall complete or partial remission response.

Adverse events (AEs) were measured from the beginning of drug administration, throughout the treatment period, until 28 days after the last dose of osimertinib. AEs were graded according to the National Cancer Institute Common Terminology Criteria for Adverse Events (CTCAE), version 4.0. If a patient experienced a CTCAE of grade 3 or higher and / or unacceptable toxicity (any grade) that was considered to be associated with osimertinib, dosing could be interrupted for up to 3 weeks. If the toxicity resolved or reverted to CTCAE grade $\leq 2$ within 3 weeks of onset, osimertinib could be restarted at the same dose ( $80 \mathrm{mg}$, daily) or a lower dose (40 $\mathrm{mg}$, daily), excluding cases with any grade of pulmonary toxicity, symptomatic corrected QT interval prolongation, or corneal ulceration. Once a dose had been reduced, it was not re-escalated at future cycles.

\section{Statistical analysis}

Patients who were eligible for this study represented a heterogeneous population with prior exposures to EGFR-TKIs and potential previous exposures to multiple lines of cytotoxic chemotherapies. We assumed the null hypothesis as ORR 30\% because most patients could be chemotherapynaïve and treated with only one prior EGFR-TKI therapy. An alternative hypothesis of ORR $60 \%$ was adopted from the AURA phase I study, which showed that ORR was 61\% among 127 T790M mutation-positive patients [2]. To prove the $60 \%$ ORR of osimertinib compared to the $30 \%$ of the null hypothesis, considering the $10 \%$ drop out rate, 19 patients were required to be enrolled in this study ( $\mathrm{H} 0$ proportion, 0.3; H1 proportion, 0.6 ; sample size, 17 ; power, 0.8 ; significance, 0.05 , one-sided).

The sensitivity of the EGFR mutation test using ctDNA 
Table 1. Baseline characteristics of all the screened subjects $(n=80)$

\begin{tabular}{|c|c|c|c|}
\hline Characteristic & $\begin{array}{l}\text { ctDNA T790M- } \\
\text { positive }(n=21)\end{array}$ & $\begin{array}{l}\text { ctDNA T790M- } \\
\text { negative }(n=59)\end{array}$ & p-value \\
\hline Age (yr) & $68.3(37.4-82.6)$ & $68.1(37.8-82.8)$ & 0.425 \\
\hline Female/Male & $15(71.4) / 6(28.6)$ & $37(62.7) / 22(37.8)$ & 0.472 \\
\hline Never smokers & $18(85.7)$ & $38(62.7)$ & 0.051 \\
\hline Histology, ADC & $21(100)$ & $59(100)$ & - \\
\hline Stage IV & $21(100)$ & $59(100)$ & - \\
\hline Brain metastasis & $11(52.4)$ & $22(37.3)$ & 0.228 \\
\hline Brain RT before screening & $7(33.3)$ & $13(22.0)$ & 0.304 \\
\hline \multicolumn{4}{|l|}{ Type of progression } \\
\hline Localized & $1(4.8)$ & $18(30.5)$ & 0.018 \\
\hline Systemic & $20(95.2)$ & $41(69.5)$ & \\
\hline No. of treatments before screening & $1(1-4)$ & $1(1-7)$ & - \\
\hline $1 / 2 / 3 / 4 / 5 / 6 / 7$ & $17 / 1 / 2 / 1 / 0 / 0 / 0$ & $37 / 17 / 2 / 1 / 0 / 1 / 1$ & \\
\hline \multicolumn{4}{|l|}{ Prior TKIs therapy } \\
\hline Gefitinib & $11(52.4)$ & $33(55.9)$ & 0.298 \\
\hline Erlotinib & $5(23.8)$ & $16(27.1)$ & \\
\hline Afatinib & $3(14.3)$ & $9(15.3)$ & \\
\hline Gefitinib and afatinib & $1(4.8)$ & 0 & \\
\hline Erlotinib and afatinib & $1(4.8)$ & $1(1.7)$ & \\
\hline \multicolumn{4}{|l|}{ Type of EGFR mutation ${ }^{\text {a) }}$} \\
\hline Exon 19 deletion & $14(66.7)$ & $28(47.5)$ & 0.130 \\
\hline Exon 21 L858R/L861Q & $4(19.0)$ & $24(40.7)$ & 0.074 \\
\hline Exon 19 deletion+exon 21 L858R & $1(4.8)$ & 0 & - \\
\hline G719X & $1(4.8)$ & $2(3.4)$ & - \\
\hline No activating mutation found & $1(4.8)$ & $5(8.5)$ & - \\
\hline \multicolumn{4}{|l|}{ Time point at screening } \\
\hline After cessation of prior EGFR-TKI & $11(52.4)$ & $39(66.1)$ & 0.265 \\
\hline During EGFR-TKI treatment & $10(47.6)$ & $20(33.9)$ & \\
\hline \multicolumn{4}{|l|}{ Re-biopsy } \\
\hline Success & $9^{\mathrm{b})}(42.9)$ & $43(72.9)$ & 0.013 \\
\hline Failure & $12(57.1)$ & $16(27.1)$ & \\
\hline
\end{tabular}

Values are presented as median (range) or number (\%). ctDNA, circulating tumor DNA; ADC, adenocarcinoma; RT, radiotherapy; TKI, tyrosine kinase inhibitor; EGFR, epidermal growth factor receptor. ${ }^{\text {a) }}$ Results of tumor genotyping at diagnosis, b) Re-biopsy after osimertinib treatment $(n=4)$, invalid for analysis $(n=5)$.

was expected to be $60 \%$ from the screening analyses that were performed in previous studies $[11,12]$. Considering the $40 \%$ detection rate of the $7790 \mathrm{M}$ mutation in real-practice tissue genotyping [15-17], the number of individuals required for screening was expected to be greater than 79 patients $(19 / 0.4 / 0.6=79)$. The sensitivity of ctDNA EGFR T790M mutation-detection methods was calculated as the percentage of ctDNA T790M-positive cases (n) among the estimated T790M-positive cases $(n+$ number of cases negative for ctDNA T790M mutation×T790M-positive rate in tumor genotyping).

According to the study protocol, the ORR was assessed in the response-evaluable (RE) patients; RE patients were defined as subjects who had received at least one dose of treatment and for whom response evaluations were available. Baseline characteristics, safety data, PFS, and DoR were analyzed in an intent-to-treat (ITT) population $(n=19)$ (Fig. 1). The expected median PFS, set to 10 months, was adopted from the AURA phase I study [2] in which the median PFS was 9.6 months (updated to 12.3 months and 10.1 months in AURA phase II and III studies, respectively) $[3,4]$.

Intergroup comparisons were performed using the MannWhitney U test for continuous variables, and Pearson's chisquare or Fisher exact tests for categorical variables. Survival times were estimated for each group using the Kaplan-Meier method. Statistical analyses were performed with IBM SPSS 
Table 2. Sensitivity of ctDNA testing for detection of T790M mutation

\begin{tabular}{|c|c|}
\hline ctDNA EGFR mutation tested screening subject & No. $(\%)(\mathrm{n}=80)$ \\
\hline T790M-positive & $21(26.3)$ \\
\hline Known activating EGFR mutation detected & $20(95.2)$ \\
\hline Activating EGFR mutation not detected ${ }^{\text {a) }}$ & $1(4.8)$ \\
\hline (1) PANA Mutyper only & 4 \\
\hline (2) Cobas EGFR mutation test version 2 only & 4 \\
\hline Both (1) and (2) & 13 \\
\hline T790M-negative & $59(73.7)$ \\
\hline Known activating EGFR mutation detected & $32(54.2)$ \\
\hline Tumor genotyping performed & 20 \\
\hline Tumor T790M-positive & $5(25.0)$ \\
\hline Known activating EGFR mutation not detected & $27(45.8)$ \\
\hline Tumor genotyping performed & 23 \\
\hline Tumor T790M-positive & $7(30.4)$ \\
\hline Estimated T790M-positive cases in screening subjects ${ }^{\text {b) }}$ & $37=21+[59 \times(12 / 43)]$ \\
\hline \multicolumn{2}{|l|}{ Sensitivity of tests } \\
\hline (1) PANA Mutyper & $17 / 37(45.9)$ \\
\hline (2) Cobas EGFR mutation test ver. 2 & $17 / 37(45.9)$ \\
\hline Combination of (1) and (2) & $21 / 37$ (56.8) \\
\hline
\end{tabular}

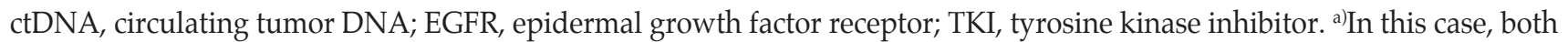
tests (1) and (2) detected T790M from ctDNA. However, no EGFR mutation was detected in tumor genotyping at diagnosis or in pleural fluid genotyping after failure of first-line EGFR-TKI treatment (case No. 10), b) As the T790M mutation was detected in 12 of 43 tumor genotyping tests, 27.9\% (12/43) of $59 \mathrm{ctDNA}$-negative cases was assumed to have T790M (n=16). If we added 21 cases with positive ctDNA T790M, a total of 37 patients were estimated to have the T790M EGFR mutation.

ver. 23 (IBM Corp., Armonk, NY), and p-values of $<0.05$ indicated statistical significance.

\section{Ethical statement}

This study was conducted in accordance with the Declaration of Helsinki and Good Clinical Practice guidelines and was approved by the institutional ethics review boards (CNUHH-2016-065) and the Korean Ministry of Food and Drug Safety (30956). All patients were required to provide written informed consent before participating in this study. The trial was registered at clinicaltrials.gov (NCT02769286).

\section{Results}

\section{Patient characteristics}

Eighty patients with acquired resistance to prior EGFRTKIs were screened for the T790M resistance mutation from September 2016 to September 2017. The majority of patients were female and never smokers. The ctDNA of 21 patients
(26.3\%) were positive for the T790M mutation, and 19 subjects were enrolled in this trial. Among 59 patients negative for the ctDNA T790M mutation, genotyping using tumor tissue or cytology samples were successful in 43 patients, and the T790M mutation was detected in 12 patients $(12 / 43$, 27.9\%) (Fig. 1).

Baseline characteristics of the screened patients according to positivity of ctDNA T790M mutation are compared in Table 1 . The ctDNA T790M-positive cases were more likely to have systemic progression $(\mathrm{p}=0.018)$, a higher rate of brain metastasis at screening $(\mathrm{p}=0.228)$, and a higher proportion of exon 19 deletion mutations $(p=0.130)$ than ctDNA T790Mnegative patients.

\section{Sensitivity of EGFR mutation tests for T790M detection}

The EGFR T790M mutation was detected by both the Pana and Cobas tests in 13 cases, only by Pana in four cases, and only by Cobas in four cases. Both T790M and EGFR sensitizing mutations were detected in $95.2 \%$ of the ctDNA T790Mpositive cases (20/21) (Table 2).

Among 59 T790M-negative ctDNA samples, the EGFRactivating mutation was present in only $54.2 \%$ of cases, suggesting false-negative results of ctDNA testing due to low 
A

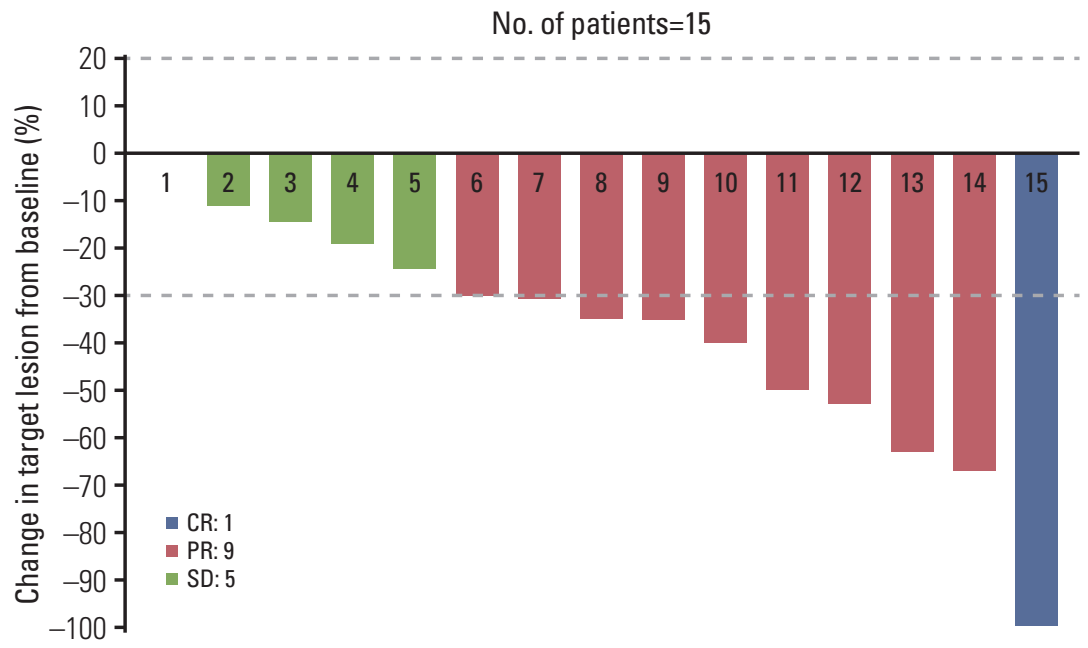

B

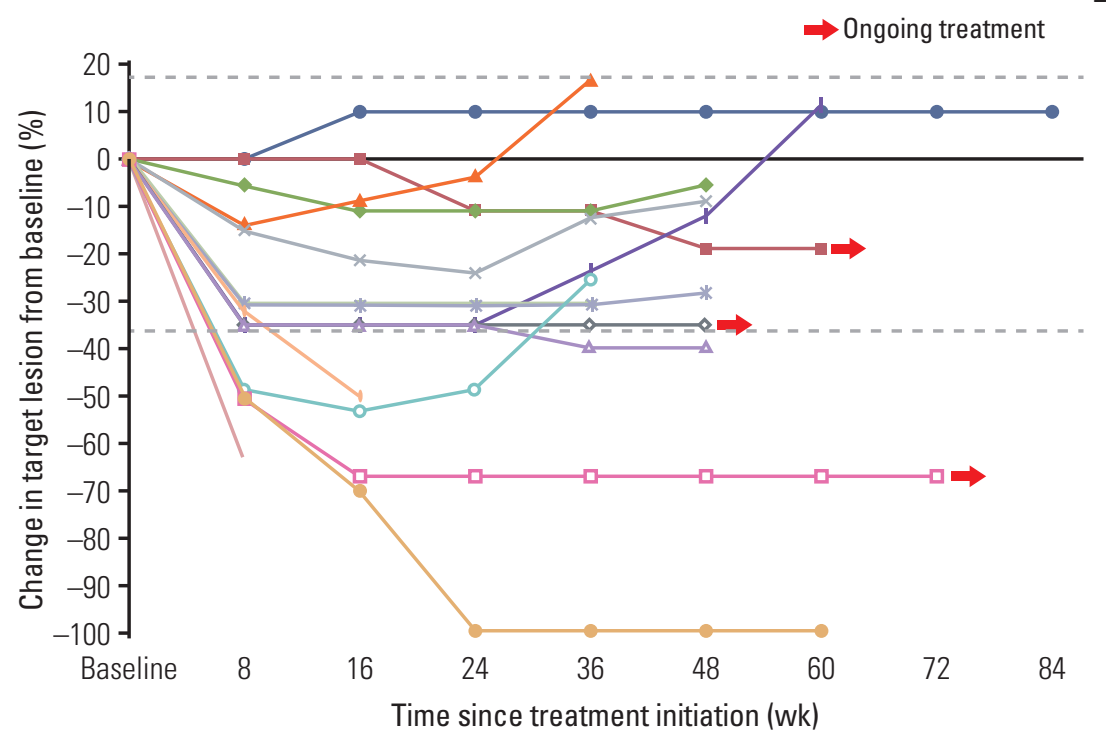

Fig. 2. Response to osimertinib. Waterfall plot (A) and spider plot (B) according to Response Evaluation Criteria in Solid Tumors in the response evaluable population $(n=15)$. CR, complete response; PR, partial response; SD, stable disease.

tumor DNA content or poor DNA quality. With subsequent genotyping tests using tumor samples acquired immediately after failure of ctDNA testing $(29 / 43)$ or one or more lines of chemotherapy (14/43), the T790M mutation was detected in 12 of 43 cases $(27.9 \%)$; thus, the estimated prevalence of the T790M mutant was calculated as $59 \times 0.279(n=16)$. If we added ctDNA T790M-positive cases $(\mathrm{n}=21)$, the total number of T790M EGFR mutants was calculated as 37 among 80 screened subjects $(46.3 \%)$. Thus, the sensitivity of the ctDNA tests was $56.8 \%(21 / 37)$ using both methods and $45.9 \%$
(17/37) with either the Pana or Cobas test (Table 2).

\section{Response and survival}

The response to osimertinib was evaluable in 15 patients (RE population). Among the RE population, complete ( $\mathrm{n}=1)$ or partial $(n=9)$ remissions were observed in 10 cases (ORR $66.7 \%$ ), and the disease control rate (DCR) was $100.0 \%$ (Fig. 2). In the ITT population, the ORR and DCR were $52.6 \%(10 / 19)$ and 78.9\% (15/19), respectively. The final analysis of PFS and 
Table 3. Clinical efficacy of osimertinib treatment according to brain metastasis

\begin{tabular}{|c|c|c|c|c|c|c|c|c|}
\hline Efficacy & $\begin{array}{c}\text { ITT } \\
(n=19)\end{array}$ & $\begin{array}{l}\text { Both Pana and } \\
\left.\text { Cobas }(n=11)^{a}\right)\end{array}$ & $\begin{array}{l}\text { Pana only } \\
\quad(n=4)\end{array}$ & $\begin{array}{l}\text { Cobas only } \\
\qquad(n=4)\end{array}$ & p-value & $\begin{array}{l}\text { Without } \\
\text { BM (n=9) }\end{array}$ & $\begin{array}{c}\text { With } \\
\text { BM (n=10) }\end{array}$ & p-value \\
\hline \multicolumn{9}{|l|}{ Type of response } \\
\hline Not evaluable & $4(21.0)$ & $2(18.2)$ & $1(25.0)$ & $1(25.0)$ & 0.702 & $2(22.2)$ & $2(20.0)$ & 0.289 \\
\hline Complete & $1(5.3)$ & $1(9.1)$ & 0 & 0 & & 0 & $1(10.0)$ & \\
\hline Partial & $9(47.4)$ & $5(45.4)$ & $3(75.0)$ & $1(25.0)$ & & $6(66.7)$ & $3(30.0)$ & \\
\hline Stable disease & $5(26.3)$ & $3(27.3)$ & 0 & $2(50.0)$ & & $1(11.1)$ & $4(40.0)$ & \\
\hline Progression & 0 & 0 & 0 & 0 & & 0 & 0 & \\
\hline ORR $^{\text {b) }}$ & $10 / 15(66.7)$ & $6 / 9(66.7)$ & $3 / 3(100)$ & $1 / 3(33.3)$ & 0.223 & $6 / 7(85.7)$ & $4 / 8(50.0)$ & 0.282 \\
\hline $\mathrm{DCR}^{\mathrm{b})}$ & $15 / 15(100)$ & $9 / 9(100)$ & $3 / 3(100)$ & $3 / 3(100)$ & - & $7 / 7(100)$ & $8 / 8(100)$ & - \\
\hline PFS (mo) & $8.3(7.9-8.7)$ & - & - & - & - & $5.1(0.0-13.9)$ & $8.4(6.5-10.3)$ & 0.431 \\
\hline TTR (mo) & $1.6(1.6-1.7)$ & - & - & - & - & $1.6(1.6-1.7)$ & $1.6(1.6-1.7)$ & 0.789 \\
\hline DoR (mo) & $6.8(5.3-8.3)$ & - & - & - & - & $6.7(0.9-12.5)$ & $6.8(1.1-12.5)$ & 0.782 \\
\hline
\end{tabular}

Values are presented as number $(\%)$ or median (95\% confidence interval). ITT, intention-to-treat population; Pana, PANA Mutyper; Cobas, Cobas EGFR mutation test ver. 2; BM, brain metastasis; ORR, objective response rate; DCR, disease control rate; PFS, progression-free survival; TTR, time to response; DoR, duration of response. ${ }^{\text {a) }}$ Thirteen patients were positive for

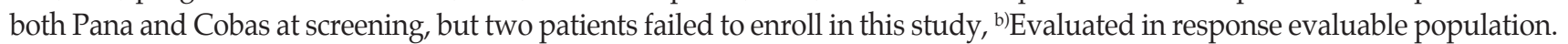

DoR was performed at $84.2 \%$ maturity $(16 / 19)$ in the ITT population. The data cut-off date was June 30, 2018 and the median follow-up duration was 15.7 months (95\% confidence interval [CI], 13.0 to 18.4). The median PFS was 8.3 months (95\% CI, 7.9 to 8.7), and the median DoR was 6.8 months (95\% CI, 5.3 to 8.3). Most patients showed objective responses within two cycles of osimertinib. In comparison to the treatment efficacy parameters, ORR and PFS according to brain metastasis were not significant (Table 3).

Response to osimertinib was not significantly different according to the ctDNA genotyping method. Excluding the patients who failed the screening $(n=2)$ and those who were incapable of undergoing the response assessment $(n=4)$, ORR and DCR according to positive results of T790M mutation tests were as follows: both Pana and Cobas positive, $67 \%$ and $100 \%$; Pana only, 100\% and 100\%; and Cobas only, 33\% and $100 \%$; respectively ( $\mathrm{p}>0.05)$ (Table 3 ).

Four patients were not assessed with respect to their responses to treatment due to poor adherence to treatment from sepsis $(n=1)$ or rapid deterioration leading to death $(n=3)$ (Table 4, Fig. 1). These four patients were treated with osimertinib for less than 2 weeks. Molecular profiles of tumor genotyping and clinical efficacy of osimertinib in all ctDNA T790M-positive patients are described in Table 4.

\section{Toxicity and safety}

Table 5 shows the toxicity profiles of the ITT population. AEs occurred in 17 of 19 patients (89.5\%), and grade 3 or 4 events developed in six patients $(31.6 \%)$. The majority of AEs were gastrointestinal and skin manifestations. One subject (numbered 3 in Table 4) experienced grade 3 drug-related AEs. With osimertinib as a second-line treatment, partial remission was observed in the first response evaluation; however, chest imaging showed diffuse ground-glass opacities and multifocal consolidations in both lungs, which were suggestive of interstitial pneumonitis. After discontinuing osimertinib therapy, the pneumonitis was treated with prednisolone and empirical antibiotic treatment. Without recontinuation of osimertinib therapy, the patient showed disease progression after 5.1 months of PFS, and then received cytotoxic chemotherapy and olmutinib.

\section{Discussion}

This is the first prospective trial evaluating the treatment efficacy of osimertinib in patients with previously EGFRTKI-treated NSCLC harboring the EGFR T790M resistance mutation, detected from ctDNA with unknown EGFR tumor mutation status. In the present study, osimertinib showed favorable efficacy and a similar safety profile, in comparison with previous trials that were based on tumor or plasma genotyping [2-4].

In a post hoc analysis of samples from the AURA phase I trial, which enrolled patients with and without tumor T790M mutations, Oxnard et al. [11] demonstrated that patients positive for T790M in their plasma showed comparable efficacy 
Table 4. Molecular profiles of tumor genotyping and clinical efficacy of osimertinib in ctDNA T790M-positive patients

\begin{tabular}{|c|c|c|c|c|c|c|c|c|c|c|}
\hline $\begin{array}{l}\text { Case } \\
\text { No. }\end{array}$ & $\begin{array}{c}\text { ctDNA } \\
\text { T790M } \\
\text { Pana/Cobas }\end{array}$ & $\begin{array}{l}\text { Known } \\
\text { activating } \\
\text { mutation }\end{array}$ & $\begin{array}{l}\text { Sources of } \\
\text { re-biopsy }\end{array}$ & $\begin{array}{c}\text { Time } \\
\text { point of } \\
\text { re-biopsya) }\end{array}$ & $\begin{array}{c}\text { Re-biopsy } \\
\text { T790M }\end{array}$ & $\begin{array}{l}\text { Re-biopsy } \\
\text { activating } \\
\text { mutation }\end{array}$ & $\begin{array}{c}\text { Prior } \\
\text { EGFR-TKI }\end{array}$ & $\begin{array}{l}\text { Treatment } \\
\text { line }\end{array}$ & $\begin{array}{c}\text { Best } \\
\text { response }\end{array}$ & $\begin{array}{l}\text { PFS } \\
(\mathrm{mo})\end{array}$ \\
\hline 1 & $+/+$ & L858R & Tissue & After & + & L858R & Afatinib & 2 & CR & 13.4 \\
\hline 2 & $+1+$ & E19del & Tissue & After & - & E19del & Erlotinib & 2 & PR & 11.0 \\
\hline 3 & $+/+$ & L858R & Tissue & Before $^{\text {b) }}$ & + & L858R & Gefitinib & 2 & PR & 5.1 \\
\hline 4 & $+1+$ & E19del & - & - & NA & - & Gefitinib & 2 & PR & Ongoing \\
\hline 5 & $+1+$ & E19del & - & - & NA & - & Gefitinib & 2 & PR & 3.7 \\
\hline 6 & $+1+$ & E19del & - & - & NA & - & Erlotinib & 2 & PR & 12.7 \\
\hline 7 & $+/+$ & E19del & Tissue & After & - & E19del & Gefitinib & 2 & SD & 8.2 \\
\hline 8 & $+1+$ & E19del & Bone marrow & Before & - & Wild & Gefitinib & 4 & SD & 12.3 \\
\hline 9 & $+/+$ & L858R & - & - & NA & - & Gefitinib & 2 & SD & Ongoing \\
\hline 10 & $+1+$ & Unknown & Pleural fluid & Before & - & Wild & Gefitinib & 3 & $\mathrm{NE}$ & 1.0 \\
\hline 11 & $+1+$ & L858R & - & - & NA & - & Gefitinib & 2 & NE & 2.5 \\
\hline 12 & $+/+$ & E19del & - & - & NA & - & Gefitinib & - & $\begin{array}{l}\text { Screening } \\
\text { fail }\end{array}$ & - \\
\hline 13 & $+/+$ & E19del & - & - & NA & - & Erlotinib & - & $\begin{array}{l}\text { Screening } \\
\text { fail }\end{array}$ & - \\
\hline 14 & $+1-$ & E19del & - & - & NA & - & $\begin{array}{c}\text { Gefitinib, } \\
\text { afatinib }\end{array}$ & 5 & PR & 8.4 \\
\hline 15 & $+1-$ & E19del & - & - & NA & - & Afatinib & 2 & PR & 9.4 \\
\hline 16 & $+1-$ & E19del L858R & - & - & NA & - & Erlotinib & 2 & PR & 8.3 \\
\hline 17 & $+/-$ & G719X & - & - & NA & - & Afatinib & 2 & $\mathrm{NE}$ & 0.3 \\
\hline 18 & $-/+$ & E19del & Tissue & Before & $\begin{array}{l}\text { Not } \\
\text { requested }\end{array}$ & $\begin{array}{l}\text { Not } \\
\text { requested }\end{array}$ & Gefitinib & 2 & PR & Ongoing \\
\hline 19 & $-1+$ & E19del & Tissue & After & + & E19del & $\begin{array}{c}\text { Gefitinib, } \\
\text { afatinib }\end{array}$ & 4 & SD & 18.5 \\
\hline 20 & $-1+$ & E19del & Tissue & Before & Invalid & Invalid & Erlotinib & 2 & SD & 9.9 \\
\hline 21 & $-1+$ & E19del & - & - & NA & - & Gefitinib & 2 & NE & 0.5 \\
\hline
\end{tabular}

ctDNA, circulating tumor DNA; Pana, PANA Mutyper; Cobas, Cobas EGFR mutation test ver. 2; EGFR, epidermal growth factor receptor; TKI, tyrosine kinase inhibitor; PFS, progression-free survival; CR, complete response; PR, partial response; $\mathrm{NA}$, not available; SD, stable disease; NE, not evaluable. a)Re-biopsy "before" or "after" administration of the third-generation

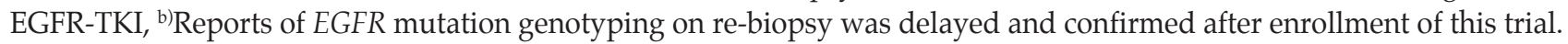

(ORR and PFS), which was similar to the results that were based on tumor genotyping. These data suggested a new paradigm: plasma genotyping could be used as a screening tool before tissue re-biopsy for the detection of acquired resistance, and a positive result obviates tumor biopsy. The difference in our study is that we prospectively enrolled patients using only plasma genotyping among patients with acquired resistance to prior EGFR-TKI, which reflects realworld practice. We have demonstrated the utility of plasma genotyping as a companion diagnostic test for osimertinib treatment.

Osimertinib showed marked penetration of the bloodbrain barrier and greater access to the brain in animal models [18]. In the AURA trials, osimertinib demonstrated meaningful efficacy against CNS metastases, including leptomenin- geal seeding [3,4,19-21]. Our results also show acceptable CNS effects, despite a greater proportion of brain metastases than the AURA 3 trial (52.6\% vs. 33\%). While the PFS in the ITT population of this trial was shorter than that of the AURA 3 trial (median, 8.3 months; $95 \%$ CI, 7.9 to 8.7 vs. 10.1 months; $95 \% \mathrm{CI}, 8.3$ to 12.3 ), the PFS in the brain metastasis subgroup was similar between the two trials (median, 8.4 months; $95 \%$ CI, 6.5 to 10.3 vs. 8.5 months; $95 \%$ CI, 6.8 to 12.3). In this trial, there were no significant differences in ORR, median PFS, and DoR according to brain metastasis. Therefore, these data suggest that the clinical efficacy of osimertinib can be maintained, irrespective of brain metastasis.

After acquiring resistance to EGFR-TKIs, testing to confirm the acquisition of the T790M mutation has become a manda- 
Table 5. Adverse events

\begin{tabular}{|c|c|c|}
\hline & Any grade (n=19) & Grade $\geq 3(n=19)$ \\
\hline Adverse events & $17(89.5)$ & $6(31.6)$ \\
\hline Drug-related adverse events & $10(52.6)$ & $1(5.3)$ \\
\hline AEs leading to dose reduction & & 0 \\
\hline AEs leading to drug discontinuation ${ }^{\text {a) }}$ & & $1(5.3)$ \\
\hline Serious adverse events & & $8(42.1)$ \\
\hline Serious adverse events, drug-related & & $1(5.3)$ \\
\hline \multicolumn{3}{|l|}{ Common events } \\
\hline Gastritis & $4(21.0)$ & 0 \\
\hline Paronychia & $4(21.0)$ & 0 \\
\hline Diarrhea & $3(15.8)$ & 0 \\
\hline Nausea & $3(15.8)$ & 0 \\
\hline Constipation & $3(15.8)$ & 0 \\
\hline Rash or Acne & $3(15.8)$ & 0 \\
\hline Headache & $3(15.8)$ & 0 \\
\hline Back pain & $3(15.8)$ & $1(5.3)$ \\
\hline Pruritus & $2(10.5)$ & 0 \\
\hline Mucositis & $2(10.5)$ & 0 \\
\hline LFT $^{\text {b) }}$ elevation & $2(10.5)$ & $1(5.3)$ \\
\hline Sepsis & $2(10.5)$ & $2(10.5)$ \\
\hline Vomiting & $1(5.3)$ & $1(5.3)$ \\
\hline Anemia & $1(5.3)$ & 0 \\
\hline Pneumonitis ${ }^{\text {a) }}$ & $1(5.3)$ & $1(5.3)$ \\
\hline
\end{tabular}

Values are presented as number (\%). LFT, liver function test. ${ }^{a}$ Interstitial pneumonia, ${ }^{\mathrm{b})}$ Aspartate aminotransferase or alanine aminotransferase.

tory procedure that is, this far, usually based on tumor genotyping. However, tumor biopsies have clear limitations in clinical practice for patients with advanced lung neoplasms [7]. In a retrospective analysis of 139 patients with prior EGFR-TKI failure, Kawamura et al. [15] found that re-biopsy was performed in only $54 \%$ (75 patients), and the remaining $46 \%$ (64 patients) could not undergo re-biopsy due to poor performance status $(n=19)$, inaccessible tumor site $(n=19)$, and other reasons $(\mathrm{n}=26)$. In this trial, we performed re-biopsy in 73\% (43 of 59) of ctDNA T790M-negative patients. However, one-third of ctDNA T790M-negative patients $(14 / 43)$ underwent re-biopsies after the failure of one or more lines of chemotherapies when their tumors progressed further.

In a prospective study using droplet digital PCR, plasma genotyping significantly shortened the median turnaround time for the detection of acquired resistance compared with tissue genotyping ( 3 days vs. 27 days), with favorable specificity $(63 \%)$ and sensitivity (77\%) [22]. Therefore, the development of plasma-based liquid biopsies has changed the care of patients with lung cancer, especially those with advancedstage disease.

Even after proving its impact on cancer care, concerns remain about introducing liquid biopsy into the clinical setting with respect to test sensitivity and false-negative or false-positive rates. The AURA trials of osimertinib showed favorable sensitivity for plasma genotyping of the T790M mutation (70\% with BEAMing [11] and 51\%-64\% with the Cobas test [4,12]). Our trial did not mandate tumor biopsy in patients who tested positive for T790M mutation on plasma analysis; thus, there is no reference to confirm true positivity or sensitivity. However, as the Cobas test was approved by the United States and European countries, we compared the results of the Cobas and Pana tests, as shown in Tables 2 and 3. In our results, two tests (Pana and Cobas) for detecting ctDNA harboring the T790M mutation showed similar and comparable sensitivity to previous studies (45.9\% respectively and $56.8 \%$ in combination) (Table 2 ).

In the AURA phase I study, Oxnard et al. [11] adverted discordance (T790M plasma-positive and tumor-negative or T79-0M plasma-negative and tumor-positive) through using the BEAMing assay, which represents a more sensitive technique than PCR. In their study, false-positives were minor $(\mathrm{n}=18)$ among the total diagnostic analysis set $(\mathrm{n}=216)$ [11]. In the AURA phase II study, the false-positive rate of the Cobas test was very low when assessed further using next- 
generation sequencing (MiSeq) [12]. The plasma T790M-negative subgroup in the AURA phase I study showed distinct differences in PFS according to tumor T790M positivity. Furthermore, in the present study, tumor biopsy was performed if the T790M mutation was not detected by plasma tests. Among the 43 patients who underwent subsequent tumor biopsy, 12 false-negative cases were detected. These 12 patients were treated with either osimertinib $(\mathrm{n}=10)$ or olmutinib $(\mathrm{n}=2)$. The ORR and DCR were 25.0\% (3/12) and 91.7\% (11/ 12), and the median PFS was 5.9 months (95\% CI, 0.0 to 13.0). Thus, tumor genotyping is necessary if plasma genotyping for T790M shows negative results, as the plasma T790M-negative population is composed of true and false-negatives.

Owing to an unknown tumor genotyping status at screening, the false-negative and false-positive rates of our study remain uncertain. However, the present plasma assay provided sufficient sensitivity to achieve the expected clinical outcomes (ORR 67\% and median PFS 8.3 months). In addition, the clinical efficacy was not significantly affected by which tests (Pana or Cobas) showed positivity for the T790M mutation $(p>0.05)$ (Table 3).

The safety profile of this trial was consistent with previous reports of AURA trials [2-5]. Osimertinib was well tolerated, and there was no need for dose reductions due to related AEs. Osimertinib treatment was discontinued in one subject owing to possible drug-induced interstitial lung injury, and the pneumonitis resolved after steroid and antibiotic treatment. The incidence of osimertinib-related lung injury was reported to be approximately $4 \%$ in prior AURA trials.

Although the study maturation was not fully achieved, the median PFS of this study (8.3 months; $95 \%$ CI, 7.9 to 8.7 ) was shorter than that of the patients who were plasma T790Mpositive in the AURA phase I study (9.7 months) [11]. In the AURA phase III trial [4], the median PFS was 10.1 months in tissue T790M-positive patients; however, it was 8.2 months in tissue and plasma T790M-positive cases. The shorter PFS might be due to the characteristics of patients with plasma T790M-positivity reflecting a high tumor burden or tumor DNA shedding features. It is possible that the patients who could not endure invasive biopsy procedures were more likely to be screened in this trial, as shown by the older patient age in this study (median 68 years vs. 60 and 62 years in the study by Oxnard et al. [11] and Mok et al. [4], respectively). In this trial, PFS was measured from the ITT population, which included four patients with poor adherence to treatment who were incapable of undergoing response evaluations.

In conclusion, osimertinib had favorable efficacy in patients with NSCLC harboring the EGFR T790M resistance mutation, which was detected from ctDNA with unknown tumor T790M mutation status. The results of this phase II prospective trial support the change from tumor biopsy to plasma ctDNA in current screening practices for detection of the T790M mutation, which is a prerequisite for the prescription of third-generation EGFR-TKI after acquiring resistance to prior EGFR-TKIs. However, if the T790M mutation is not detected from the ctDNA, we recommend tumor biopsy, as ctDNA screening tests may present false-negative results.

\section{Electronic Supplementary Material}

Supplementary materials are available at Cancer Research and Treatment website (https:// www.e-crt.org).

\section{Conflicts of Interest}

The current study was financially supported by AstraZeneca, Panagene, and Roche Molecular Diagnostics, Korea. However, the funders had no role in the study design, data collection and analysis, decision to publish, or preparation of this manuscript.

\section{References}

1. Yu HA, Arcila ME, Rekhtman N, Sima CS, Zakowski MF, Pao $\mathrm{W}$, et al. Analysis of tumor specimens at the time of acquired resistance to EGFR-TKI therapy in 155 patients with EGFRmutant lung cancers. Clin Cancer Res. 2013;19:2240-7.

2. Janne PA, Yang JC, Kim DW, Planchard D, Ohe Y, Ramalingam SS, et al. AZD9291 in EGFR inhibitor-resistant nonsmall-cell lung cancer. N Engl J Med. 2015;372:1689-99.

3. Yang JC, Ahn MJ, Kim DW, Ramalingam SS, Sequist LV, Su WC, et al. Osimertinib in pretreated T790M-positive advanced non-small-cell lung cancer: AURA study phase II extension component. J Clin Oncol. 2017;35:1288-96.

4. Mok TS, Wu YL, Ahn MJ, Garassino MC, Kim HR, Rama- lingam SS, et al. Osimertinib or platinum-pemetrexed in EGFR T790M-positive lung cancer. N Engl J Med. 2017;376:629-40.

5. Soria JC, Ohe Y, Vansteenkiste J, Reungwetwattana T, Chewaskulyong B, Lee $\mathrm{KH}$, et al. Osimertinib in untreated EGFR-mutated advanced non-small-cell lung cancer. N Engl J Med. 2018;378:113-25.

6. Ricciuti B, Baglivo S, Paglialunga L, De Giglio A, Bellezza G, Chiari $\mathrm{R}$, et al. Osimertinib in patients with advanced epidermal growth factor receptor T790M mutation-positive nonsmall cell lung cancer: rationale, evidence and place in therapy. Ther Adv Med Oncol. 2017;9:387-404.

7. Crowley E, Di Nicolantonio F, Loupakis F, Bardelli A. Liquid 
biopsy: monitoring cancer-genetics in the blood. Nat Rev Clin Oncol. 2013;10:472-84.

8. Douillard JY, Ostoros G, Cobo M, Ciuleanu T, Cole R, McWalter $\mathrm{G}$, et al. Gefitinib treatment in EGFR mutated caucasian NSCLC: circulating-free tumor DNA as a surrogate for determination of EGFR status. J Thorac Oncol. 2014;9:1345-53.

9. Qiu M, Wang J, Xu Y, Ding X, Li M, Jiang F, et al. Circulating tumor DNA is effective for the detection of EGFR mutation in non-small cell lung cancer: a meta-analysis. Cancer Epidemiol Biomarkers Prev. 2015;24:206-12.

10. Thress KS, Brant R, Carr TH, Dearden S, Jenkins S, Brown H, et al. EGFR mutation detection in ctDNA from NSCLC patient plasma: a cross-platform comparison of leading technologies to support the clinical development of AZD9291. Lung Cancer. 2015;90:509-15.

11. Oxnard GR, Thress KS, Alden RS, Lawrance R, Paweletz CP, Cantarini M, et al. Association between plasma genotyping and outcomes of treatment with osimertinib (AZD9291) in advanced non-small-cell lung cancer. J Clin Oncol. 2016;34: 3375-82.

12. Jenkins S, Yang JC, Ramalingam SS, Yu K, Patel S, Weston S, et al. Plasma ctDNA Analysis for detection of the EGFR T790M mutation in patients with advanced non-small cell lung cancer. J Thorac Oncol. 2017;12:1061-70.

13. Jackman D, Pao W, Riely GJ, Engelman JA, Kris MG, Janne PA, et al. Clinical definition of acquired resistance to epidermal growth factor receptor tyrosine kinase inhibitors in nonsmall-cell lung cancer. J Clin Oncol. 2010;28:357-60.

14. Eisenhauer EA, Therasse P, Bogaerts J, Schwartz LH, Sargent D, Ford R, et al. New response evaluation criteria in solid tumours: revised RECIST guideline (version 1.1). Eur J Cancer. 2009;45:228-47.

15. Kawamura T, Kenmotsu H, Taira T, Omori S, Nakashima K,
Wakuda K, et al. Rebiopsy for patients with non-small-cell lung cancer after epidermal growth factor receptor-tyrosine kinase inhibitor failure. Cancer Sci. 2016;107:1001-5.

16. Sundaresan TK, Sequist LV, Heymach JV, Riely GJ, Janne PA, Koch WH, et al. Detection of T790M, the acquired resistance EGFR mutation, by tumor biopsy versus noninvasive bloodbased analyses. Clin Cancer Res. 2016;22:1103-10.

17. Kawamura T, Kenmotsu H, Omori S, Nakashima K, Wakuda $\mathrm{K}$, Ono A, et al. Clinical factors predicting detection of T790M mutation in rebiopsy for EGFR-mutant non-small-cell lung cancer. Clin Lung Cancer. 2018;19:e247-52.

18. Ballard P, Yates JW, Yang Z, Kim DW, Yang JC, Cantarini M, et al. Preclinical comparison of osimertinib with other EGFRTKIs in EGFR-mutant NSCLC brain metastases models, and early evidence of clinical brain metastases activity. Clin Cancer Res. 2016;22:5130-40.

19. Goss G, Tsai CM, Shepherd FA, Ahn MJ, Bazhenova L, Crino $\mathrm{L}$, et al. CNS response to osimertinib in patients with T790Mpositive advanced NSCLC: pooled data from two phase II trials. Ann Oncol. 2018;29:687-93.

20. Mok T, Ahn MJ, Han JY, Kang JH, Katakami N, Kim H, et al. CNS response to osimertinib in patients (pts) with T790M-positive advanced NSCLC: data from a randomized phase III trial (AURA3). J Clin Oncol. 2017;35(15 Suppl):9005.

21. Yang JC, Kim DW, Kim SW, Cho BC, Lee JS, Ye X, et al. Osimertinib activity in patients (pts) with leptomeningeal (LM) disease from non-small cell lung cancer (NSCLC): updated results from BLOOM, a phase I study. J Clin Oncol. 2016;34(15 Suppl):9002.

22. Sacher AG, Paweletz C, Dahlberg SE, Alden RS, O'Connell A, Feeney N, et al. Prospective validation of rapid plasma genotyping for the detection of EGFR and KRAS mutations in advanced lung cancer. JAMA Oncol. 2016;2:1014-22. 